\title{
Bronquiectasias secundarias a Tuberculosis pulmonar en pacientes de un hospital general
}

\author{
Bronchiectasis secondary to pulmonary tuberculosis in patients in a general hospital \\ Félix Llanos-Tejada ${ }^{1, a ; 2, b}$, Roberto Tamayo-Alarcón ${ }^{2, c}$
}

\section{RESUMEN}

La Tuberculosis pulmonar (TBP) supone un problema de Salud Pública. Se estima que la mitad de los pacientes con TBP desarrollan a lo largo de su vida una complicación, siendo la más frecuente la bronquiectasia. Objetivos: Determinar las principales características epidemiológicas y clínicas de pacientes con bronquiectasias secundarias a TBP en un hospital general. Material y métodos: Estudio observacional, retrospectivo, descriptivo, transversal. Se revisaron las historias clínicas de los casos con diagnóstico de bronquiectasia secundaria a TBP. Resultados: El $69 \%$ fue de sexo femenino y $40 \%$ fue adulto mayor. Las manifestaciones clínicas más frecuentes fueron tos en $66,6 \%$, expectoración 56,0\% y hemoptisis 49,2\%. El 43\% tuvo patrón tomográfico sacular. El 53\% recibió broncodilatadores, $41 \%$ corticoides inhalados, 33\% antibióticos y 16\% mucolíticos. El tiempo promedio entre el diagnóstico de TBP y el desarrollo de la bronquiectasia fue 16,76 $\pm 11,94$ años. Conclusiones: Las bronquiectasias por TBP fueron más frecuentes en mujeres y en adultos mayores. Las manifestaciones más frecuentes fueron tos, expectoración y hemoptisis. El patrón tomográfico más frecuente fue el sacular. El tratamiento más frecuente fue el uso de broncodilatadores.

PALABRAS CLAVE: Tuberculosis, bronquiectasia, epidemiología, signos y síntomas. (Fuente: DeCS Bireme).

\section{SUMMARY}

Pulmonary tuberculosis (PT) is a public health problem, it is estimated that patients with PT developed one complication during life with bronchiectasis being the most frequent. Objectives: Ton describe clinical and epidemiologic features of patients with bronchiectasis secondary to PT in a general hospital in Lima. Methods: A cross-sectional study was conducted in which medical reports of patients with bronchiectasis secondary to PT were reviewed. Results: $69 \%$ of patients were female; $40 \%$ were elder. Most frequent clinical manifestations were cough in $66.6 \%$, expectoration in $56.0 \%$ and hemoptysis in $49.2 \%$. Saccular tomographic pattern was observed in $43 \%$. Bronchodilators were prescribed for 53\%, 41\% received inhaled bronchodilators, 33 received antibiotics and 16\% mucolytic medications. Mean time from diagnosis of PT to development of bronchiectasis was $16.76 \pm 11.94$ years. Conclusions: Bronchiectasis secondary to PT were more common in females and in the elder population. The most common clinical manifestations were cough, expectoration and hemoptysis. The most common pattern at CT scan was the saccular and bronchodilators were the most common drugs used.

KEYWORDS: Tuberculosis, bronchiectasis, epidemiology, signs and symptoms. (Source: MeSH NLM).

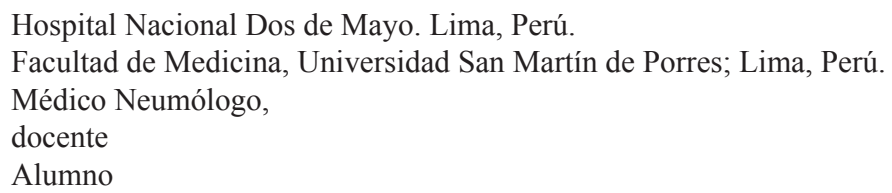




\section{INTRODUCCIÓN}

La Tuberculosis (TB) es un problema de Salud Pública a nivel mundial, de acuerdo a información de la Organización Mundial de la Salud (OMS), en el 2015, más de 10 millones en el mundo padecieron de esta enfermedad y 1,8 millones murieron debido a ella, básicamente en países con bajos y medianos ingresos (1).

Aproximadamente el $50 \%$ de los pacientes con TB pulmonar (TBP) desarrollarán alguna secuela como complicación (2). Las características biológicas del Mycobacterium tuberculosis, agente causal de la TB, asociado a la fisiopatología de la enfermedad, produce la alteración de los tejidos que conlleva a la aparición de secuelas que pueden afectar la vía aérea, el parénquima pulmonar, el sistema vascular, entre otras estructuras.

Una de las principales secuelas pulmonares secundarias a TB son las bronquiectasias (3), dilataciones anormales e irreversibles de las vías respiratorias subsiguientes a un círculo vicioso compuesto por mecanismos de defensa local ante un agente infeccioso y la inflamación de la vía respiratoria (4); lo que se asocia con una destrucción de los componentes elásticos y musculares de las paredes de dichas estructuras. Las bronquiectasias pueden ser congénitas o adquiridas, y estas últimas se asocian a un agente etiológico subyacente (5).

Hoy en día, debido a la escasa tasa de estudios que determinan su prevalencia real, parece ser más probable que en el tercer mundo y en áreas deprimidas aún siga siendo una causa importante de morbimortalidad. En el mundo industrializado, la aparición de la tomografía axial computarizada, el resurgimiento de nuevas enfermedades, como la TBP en la epidemia del SIDA o en la era del trasplante de órganos y el tratamiento supresor, y por último, la creciente longevidad de la población han generado que la prevalencia actual de las Bronquiectasias probablemente sea mayor de la esperada (6).

Impresiona que la enfermedad sea más frecuente en el sexo femenino y en la sexta década de la vida, con un mayor compromiso de los lóbulos inferiores del pulmón. Las manifestaciones clínicas más relevantes son la tos crónica, la expectoración voluminosa (que puede llegar a ser fétida o purulenta), fiebre, hemoptisis y disnea en pacientes con compromiso ventilatorio. El Gold-estándar para el diagnóstico de las bronquiectasias es la Tomografía Axial Computarizada de Alta Resolución (TAC-AR), mientras que no son las suficientemente sensibles la radiografía de tórax, la fibrobroncoscopia y la tomografía convencional (7).

En Perú, en pacientes con TBP curada se ha reportado que la principal secuela que desarrollan son las bronquiectasias (8).

El propósito del manejo en estos pacientes es reducir el número de exacerbaciones y darles una mejor calidad de vida. El tratamiento incluye el uso de antibióticos, broncodilatadores, corticoides inhalados, inmunizaciones y en algunos casos la fisioterapia respiratoria (6).

Siendo las bronquiectasias una secuela frecuente en pacientes con tuberculosis, considerando que la tuberculosis es un problema de salud pública, es importante conocer las características clínicas y epidemiológicas de las personas afectadas con esta enfermedad.

El objetivo del trabajo fue determinar las principales características clínicas y epidemiológicas de los pacientes con el diagnóstico de bronquiectasias por secuela de TBP atendidos en un hospital general.

\section{MATERIAL Y MÉTODOS}

Estudio descriptivo, transversal y retrospectivo. Se evaluaron a todos los casos con bronquiectasias que se atendieron en el año 2015, procediendo a la recolección de datos a través de la revisión de historias clínicas de los pacientes del Hospital Dos de Mayo que cumplían con los criterios de inclusión: bronquiectasias secundarias a TBP, con TEM de tórax e historia clínica completa.

Lascaracterísticas epidemiológicas que seevaluaron fueron: edad, sexo y lugar de procedencia; y las características clínicas fueron: tiempo de enfermedad, síntomas que presentó el paciente para su diagnóstico clínico, patrón tomográfico de bronquiectasia, tratamiento recibido y tiempo de demora entre la TBP y el desarrollo de las bronquiectasias.

Se utilizó el programa Microsoft Excel 2016 para procesar los datos a través de tablas simples. Se codificaron las variables cualitativas con el fin de facilitar el posterior análisis con el programa SPSS v 23. Se realizó estadística descriptiva determinando frecuencias absolutas y relativas para las variables 
categóricas y medianas, medias y desviación estándar para las variables cuantitativas. Asimismo, se utilizaron la prueba $\mathrm{T}$ de Student para comparación de muestras simples y de medias para muestras independientes con homogeneidad de varianzas y test de Chi-cuadrado de Pearson para la comparación de variables cualitativas nominales.

El estudio cumplió con los lineamientos establecidos por el Comité de Ética de la Universidad San Martín de Porres, así como los la Oficina de Apoyo a la Docencia, Capacitación e Investigación (OACDI) del Hospital Nacional Dos de Mayo.

\section{RESULTADOS}

De un total de 96 pacientes atendidos en el año 2015 con el diagnóstico de bronquiectasias (código CIE-10: J47), de acuerdo a la Oficina de Estadísticas del hospital, 65 pacientes cumplieron los criterios de inclusión, y fueron incluidos en el estudio.

Treintainueve $(60 \%)$ pacientes fueron del sexo femenino. La edad promedio fue 56,82 $\pm 17,89$ años (22 a 86 años); en mujeres fue $60,23 \pm 15,38$ años y en varones, $51,69 \pm 20,36$ años. No hubo diferencias significativas en el promedio de edad entre hombres y mujeres.

El tiempo desde el diagnóstico de TBP hasta la ocurrencia del cuadro clínico de la bronquiectasia fue 16,76 $\pm 11,94$ años ( 2 a 48 años), y el tiempo de enfermedad de la bronquiectasia en promedio fue $16,82 \pm 8,46$ años. No hubo diferencia estadística entre hombres y mujeres en ambas variables. La tos con o sin expectoración fue el síntoma más frecuente, tanto en hombres como en mujeres (Tabla 1). Sin embargo, en los adultos jóvenes fue más frecuente la hemoptisis (Tabla 2).

De acuerdo al patrón tomográfico, la bronquiectasia más frecuente fue la variante quística o sacular (43\%) (Tabla 3), seguido del patrón varicoso y cilíndrico.

El tratamiento fue principalmente con broncodilatadores en $53 \%$ de los pacientes, otros tratamientos incluyeron el uso de corticoides inhalados en $41 \%$, antibióticos crónicos (como Azitromicina) en $33 \%$ y mucolíticos en $16 \%$ de los pacientes.

Tabla 1. Características clínicas de pacientes con bronquiectasias secundarias a

Tuberculosis pulmonar, según sexo.

\begin{tabular}{lcccccc}
\hline Síntomas & \multicolumn{2}{c}{ Masculino } & \multicolumn{2}{c}{ Femenino } & \multicolumn{2}{c}{ Total } \\
\hline Tos & 15 & $57,7 \%$ & 28 & $71,8 \%$ & 43 & $66,2 \%$ \\
Disnea & 2 & $7,7 \%$ & 12 & $30,7 \%$ & 14 & $21,5 \%$ \\
Dolor torácico & 4 & $15,4 \%$ & 8 & $20,5 \%$ & 12 & $18,5 \%$ \\
Expectoración & 11 & $42,3 \%$ & 26 & $66,7 \%$ & 37 & $56,9 \%$ \\
Hemoptisis & 14 & $53,8 \%$ & 19 & $48,7 \%$ & 32 & $49,2 \%$ \\
Hiporexia & 4 & $15,4 \%$ & 5 & $12,8 \%$ & 9 & $13,8 \%$ \\
\hline
\end{tabular}

Tabla 2. Características clínicas de bronquiectasias secundarias a Tuberculosis por grupo de edad.

\begin{tabular}{lcccccc}
\hline & \multicolumn{7}{c}{ Grupo Etario } \\
\cline { 2 - 7 } & $\begin{array}{l}\text { Adulto Joven } \\
(\mathbf{1 8}-\mathbf{2 9} \text { años) }\end{array}$ & $\begin{array}{c}\text { Adulto Medio } \\
\mathbf{( 3 0}-\mathbf{6 4} \text { años) }\end{array}$ & $\begin{array}{c}\text { Adulto Mayor } \\
(>\mathbf{6 5} \text { años) }\end{array}$ \\
\hline \multicolumn{1}{c}{ Síntomas } & $\mathrm{n}(12)$ & $\%$ & $\mathrm{n}(24)$ & $\%$ & $\mathrm{n}(26)$ & $\%$ \\
Tos & 6 & $50,0 \%$ & 19 & $79,2 \%$ & 18 & $69,2-\%$ \\
Disnea & 1 & $8,3 \%$ & 7 & $29,2 \%$ & 6 & $23,1 \%$ \\
Dolor torácico & 1 & $8,3 \%$ & 5 & $20,8 \%$ & 6 & $23,1 \%$ \\
Expectoración & 7 & $58,3 \%$ & 16 & $66,7 \%$ & 14 & $53,8-\%$ \\
Hemoptisis & 7 & $58,3 \%$ & 15 & $62,5 \%$ & 10 & $38,4-\%$ \\
Hiporexia & 3 & $25,0 \%$ & 1 & $4,2 \%$ & 5 & $19,3-\%$ \\
\hline
\end{tabular}


Tabla 3. Patrones Tomográficos de bronquiectasias secundarias a tuberculosis pulmonar, según sexo.

\begin{tabular}{lcccc}
\hline Patrones tomográficos & Masculino & Femenino & \multicolumn{2}{c}{ Total } \\
\hline Quístico o sacular & 15 & 13 & 28 & $44,4 \%$ \\
Varicosa & 10 & 11 & 21 & $33,3 \%$ \\
Cilíndrico & 8 & 6 & 14 & $22,2 \%$ \\
\hline
\end{tabular}

\section{DISCUSIÓN}

El estudio se realizó para determinar las principales características epidemiológicas y clínicas de los pacientes con diagnóstico de bronquiectasias secundarias a tuberculosis pulmonar atendidos durante el año 2015, en el Hospital Nacional Dos de Mayo. Las características epidemiológicas evaluadas fueron edad, sexo y lugar de procedencia; y las características clínicas, tiempo de enfermedad, síntomas al momento del diagnóstico clínico, patrón tomográfico de bronquiectasia, tratamiento recibido y tiempo entre la TBP y el desarrollo de las bronquiectasias.

Otros estudios similares al nuestro (9-13), no incluyeron únicamente bronquiectasias por secuela de TBP (incluyeron aspergilosis broncopulmonar alérgica, fibrosis quística, procesos infecciosos recurrentes, discinesia ciliar primaria, entre otros) y algunas no consideraron exclusivamente el diagnóstico tomográfico (5).

La población más frecuentemente afectada en nuestro estudio fue el sexo femenino, lo que coincide con lo descrito previamente por otros estudios $(9,11$ 13). Sin embargo, Cereceda (10) en Concepción, Chile, en un estudio descriptivo de prevalencia obtuvo un mayor número de varones, aunque con una muestra muy pequeña, 18 en total.

La edad promedio de los pacientes en nuestro trabajo fue 56,82 $\pm 17,89$ años, aproximadamente siete años mayor al promedio descrito en otros estudios (913). Asimismo, tenemos una frecuencia alta de adultos mayores, en contraste con otros estudios donde el grupo etario más afectado fue el adulto medio. La edad se asocia a un mayor riesgo de desarrollar bronquiectasias debido a una falla en la defensa local de las vías aéreas (5). Como dato curioso, el paciente más joven, 22 años, provenía del Callao y estaba en tratamiento antirretroviral por diagnóstico de SIDA; mientras que el paciente con mayor edad, 82 años, tenía diagnóstico de EPOC moderado, diagnóstico por pruebas de función de pulmonar.
El tiempo de enfermedad o cuadro clínico al acudir a la evaluación por el Neumólogo, tuvo una media de 16,82 meses, es decir, alrededor de un año y 4 meses. Este tiempo de enfermedad quizás se pueda explicar por las evaluaciones del sintomático respiratorio en el nivel I de Atención Primaria, que busca descartar tuberculosis. Otra posible explicación es debido a que los pacientes demoran en consultar al considerar las molestias o síntomas como normales.

El síntoma más frecuente fue la tos con o sin expectoración, y luego la hemoptisis. Nuestros resultados presentan ciertas similitudes con los de otros autores; sin embargo, Gomes (9), encontró la hemoptisis como principal manifestación clínica en pacientes con bronquiectasias con $74,2 \%$ de casos. Santos (11), Newton Santos (12) y Ribeiro (13) describen a la disnea como la tercera manifestación clínica más frecuente, mientras que en nuestro estudio se presentó en menos de la cuarta parte de los pacientes.

Otros autores describen a la tos seca en casi todos los pacientes (entre $91-100 \%$ en cuatro estudios distintos), mientras que en nuestro estudio se encontró dicho síntoma, solo en dos tercios de pacientes.

En nuestro estudio, hemoptisis fue la tercera manifestación más frecuente, presente en la mitad de los pacientes; Santos (11) y Newton Santos (12), en sus estudios, determinaron que la hemoptisis no era una manifestación frecuente en los pacientes con bronquiectasias.

Nos llama la atención que en adultos jóvenes (entre 18 y 30 años), la principal manifestación clínica fue hemoptisis, en más de la mitad de los participantes. En el adulto medio (entre 30 y 65 años), en cambio, el síntoma más frecuente fue tos con expectoración; resultado similar al encontrado por Newton Santos (12). El grupo adulto mayor, fue el grupo etario más frecuente en nuestro estudio, siendo la tos y expectoración sus síntomas principales, pero además, aproximadamente $20 \%$ de los casos, presentó hiporexia. 
El patrón tomográfico más frecuente en los estudios publicados, es el de bronquiectasias cilíndricas. En nuestro estudio, sólo representó $21 \%$ de los casos, siendo más frecuentes en las bases, especialmente en hemitórax derecho. Esto quizás se pueda deber a que al estar las bronquiectasias cilíndricas mínimamente dilatadas, no suelen presentar síntomas, siendo estos los que motivan a los pacientes a acudir a la consulta médica.

En nuestro estudio, el tipo tomográfico más frecuente fue la bronquiectasia sacular o quística, (43\%). Este tipo de bronquiectasias es la expresión más grave de la enfermedad, pues adquieren una forma de saco que pueden presentar niveles hidroaéreos; adoptando, una típica imagen de "nido de golondrina" $(14,15)$, cuando el moco se acumula dentro de las lesiones. Rilance (16), afirma que es más frecuente encontrar bronquiectasias en los sitios donde se produjo la reactivación de la TBP. En nuestro estudio, las bronquiectasias saculares se ubicaron preferentemente en el hemitórax izquierdo $(60 \%$ de los casos); siendo frecuentes en el lóbulo superior ( $80 \%$ de los casos), a predominio de los segmentos posteriores.

De acuerdo a las recomendaciones de la Sociedad Británica de Tórax, el manejo está dirigido a controlar la infección, a reducir la inflamación y a promover un ambiente higiénico favorable en los bronquios (17). Asimismo, Barker (6) sostiene que la piedra angular del manejo y prevención de exacerbaciones de las bronquiectasias, son los antibióticos, los agentes mucolíticos y las técnicas de aclaramiento aéreo y de limpieza. También es frecuente el uso de broncodilatadores, antiinflamatorios, corticoides por vía inhalada e inmunizaciones.

En cuanto a nuestro estudio, el 53\% de los pacientes recibieron broncodilatadores inhalados para el cuadro de exacerbación de bronquiectasias, especialmente el salbutamol. En segundo lugar, se utilizaron corticoides inhalados, principalmente propionato de fluticasona y la budesonida.

Se utilizaron antibióticos en los pacientes que presentaron crépitos en el examen físico, aún con esputo negativo. El antibiótico de elección fue amoxicilina, pero también se utilizó levofloxacino y claritromicina. La tercera parte de los pacientes de nuestro estudio recibió al menos uno de los tres antibióticos descritos. Wong (18), realizó un estudio randomizado doble ciego en el que concluyó que los macrólidos (en especial la Azitromicina) eran una muy buena opción para la prevención de exacerbaciones en pacientes con bronquiectasias no asociadas a fibrosis quística.

Finalmente, el uso de mucolíticos estuvo presente en el $16 \%$ de casos, en todos el mucolítico fue la $\mathrm{N}$-acetil cisteína. Los estudios sobre agentes mucolíticos tienen resultados variables (19), sin embargo, la acetil cisteína no ha demostrado beneficios claros entre los pacientes con bronquiectasias no asociadas a fibrosis quística.

El periodo de años o demora entre el episodio de la TB y la aparición de síntomas sugerentes de bronquiectasias, en nuestro estudio fue de 16,76 $\pm 11,94$ años, lo que podría significar que este es el tiempo requerido para que los factores que condicionan una bronquiectasia (un agente causal, alteraciones en la arquitectura bronquial y falla en la defensa local) generen la aparición de síntomas respiratorios.

El estudio presenta las siguientes limitaciones: En primer lugar, se dejaron de evaluar historias clínicas por estar incompletas o con letra ilegible; en segundo lugar, reconocemos que por el tamaño de muestra no puede ser extrapolado a una población mayor. Sin embargo, una fortaleza fue que solo se incluyeron pacientes con bronquiectasias secundarias a TBP. A su vez, este es el primer estudio de evaluación clínica radiológica de bronquiectasias en el Hospital Nacional Dos de Mayo, que nos permite tener un panorama actual más claro de esta patología.

Así, podemos concluir, que las bronquiectasias es más frecuente en mujeres, adultos mayores de 50 años, con un tiempo de enfermedad que supera el año. Asimismo, es más frecuente la variante quística o sacular en tomografía y el síntoma más frecuente es tos con expectoración. El tratamiento más frecuente fue el uso de broncodilatadores.

\section{Declaración de financiamiento y de conflictos de interés:}

El estudio fue financiado por los autores, quienes declaran no tener conflictos de interés.

\section{Contribución de autoría:}

FLT: Diseño del estudio, interpretación de los resultados, revisión crítica del artículo y aprobación de versión final a ser publicada. RTA: Recolección y 
registro de los datos, interpretación de los resultados y redacción del artículo.

\section{Correspondencia:}

Felix Llanos Tejada

Correo electrónico: neumofekollate@aol.com

\section{REFERENCIAS BIBLIOGRÁFICAS}

1. Organización Mundial de Salud. Informe Mundial de la Tuberculosis. Ginebra: Organización Mundial de Salud; 2012

2. Romero M, Romero S, Sánchez K, SantamariaAlza Y, Mendoza T, Bolivar F. Secuelas estructurales y funcionales de tuberculosis pulmonar: una revisión de tema. RAMR. 2016; 2: 163-169.

3. Machida K, Maekura R. State of the art: Sequelae of tuberculosis. Kekkaku. 2005; 80(10): 655-74.

4. Ocampo ML, Salmón JA, Noguera VD, Zabala OC. Bronquiectasias: revisión bibliográfica. Rev posgrado VIa Cátedra Med. 2008; 182:16-9.

5. Harrison T, Kasper D. Harrison. Edicion 18. Ciudad de México: McGraw-Hill; 2014.

6. Barker A. Treatment of bronchiectasis in adults. Illinois: Uptodate; 2018. URL: https://www.uptodate. com/contents/treatment-of-bronchiectasis-in-adults

7. Chacon M. Estudio de los Pacientes con Diagnóstico de Bronquiectasias, que siguen Control en el Servicio de Neumología del Hospital Son Llátzer. Palma de Mallorca. Tesis Doctoral. Barcelona: Departamento de Medicina, Universidad Autónoma de Barcelona; 2010. 49 págs.

8. Molina G, Resurrección V, Uribe A, Figueroa M. Bronquiectasias y limitación funcional en la tuberculosis pulmonar curada. An Fac Med. 2000; 61(4): 309-14.

9. Gomes A, Medeiros M, Gifoni J. Bronquiectasia localizada e multissegmentar: perfil clínicoepidemiológico e resultado do tratamento cirúrgico em 67 casos. J Pneumologia. 2001; 27(1):1-6.
10. Cereceda J, Samzo C, Segura A, Sanhueza P. Bronquiectasias en adultos. Características clínicas. Experiencia de 5 años: 1998-2003. Rev Chil Enf Respir. 2005; 21(3): 171-78

11. Santos N, Carvalho L, Malosa L, Oliveira L.V. Características clínicas de pacientes portadores de bronquiectasias en un paciente ambulatorio. ConScientiae Saúde, 2011; 10(2):299-304.

12. Newton J, Bigatao A Santos S Studart F, Jardim J, Oliveira L. Clinical significance in non-cystic fibrosis bronquiectasis followed in a real practice. Fisioter mov. 2013; 26(4): 895-904.

13. Ribeiro F, Menezes A, Damasceno M. Evolução e perfil clínico-epidemiológico de pessoas com bronquiectasia atendidos no setor de fisioterapia ambulatorial. $\mathrm{R}$ Interd. 2014; 7(4): 157-163.

14. Reiff DB, Wells AU, Carr D, et al. CT Findings in bronchiectasis: Limited Value in Distinguishing Between Idiopathic and Specific Types. AJR Am J Roentgenol. 1995; 165 (2): 261-7.

15. Bedi P, Chalmers JD, Goeminne PC, et al. The BRICS (Bronchiectasis Radiologically Indexed CT Score): A multicenter study score for use in idiopathic and postinfective Bronchiectasis. Chest. 2017; 153 (5): 1177-1186. DOI: 10.1016/j.chest.2017.11.033

16. Rilance AB, Gerstl B. Bronchiectasis secondary to pulmonary tuberculosis. Am Rev Tuberc. 1943; 48:824.

17. Pasteur MC, Helliwell SM, Houghton SJ, Webb SC. An investigation into causative factors in patients with bronchiectasis. Am J Respir Crit Care Med. 2000; 162 (4): 1277-1284.

18. Wong C, Jayaram L, Karalus N, et al. Azithromycin for prevention of exacerbations in non-cystic fibrosis bronchiectasis (EMBRACE): a randomised, doubleblind, placebo-controlled trial. Lancet. 2012; 18 (380): 660-667.

19. Wilkinson M, Sugumar K, Milan SJ, Hart A, Crockett A, Crossingham I. Mucolytics for bronchiectasis. Cochrane Database Syst Rev. 2014; 5. doi: 10.1002/14651858.CD001289.pub2. URL: https://www.cochranelibrary.com/cdsr/ doi/10.1002/14651858.CD001289.pub2/full

Recibido: 04/04/2018

Aceptado: 28/09/2018 